DOI: $10.2478 / \mathrm{v} 10235-011-0011-4$

Szymon Wróbel

\title{
Rethinking language faculty. Has language evolved for other than language related reasons?
}

\section{Language, language faculty, language faculty in narrow sense}

The aim of this paper is to think over again the meaning of the language faculty and to provoke a discussion in order to define to what extent this concept allows to: (1) better understand the processes of the genesis of linguistic competence, (2) precisely define the conceptual apparatus of contemporary psycholinguistics in order to break through the intuitive approach to concepts, (3) give answer to the question of a more precise reconstruction of the pattern of evolution which led to the origin of language, and (4) provide answers concerning the language architecture, that is, which project of linguistic machinery comprised in the language faculty is the most adequate for description and the most fertile in terms of providing explanations.

More precisely, I will be interested in three concepts - language, faculty of language in the broad sense (FLB), and faculty of language in the narrow sense (FLN). Adopting the terminology of Mark Hauser, Noam Chomsky and Tecumseh Fitch from their famous article published in 2002, "The faculty of language: What is it, who has it, and how did it evolve?" (Hauser, Chomsky \& Fitch 2002), ${ }^{1}$ I will be considering whether the replacement

In this article I am making numerous references to the article of Mark Hauser, Noam Chomsky and Tecumseh Fitch published in Science, November 2002 (Hauser, Chomsky \& Fitch 2002). I believe there are reasons to claim that this text is of a significant importance. First, it is thanks to Chomsky's undisputed authority among language evolutionists and his so far negative comments on the evolution of language (or at least the adaptation theory), which brought a rather fierce critique. Secondly, it is because of its potential; despite its concise form, the article aims not only to provide support to the research of evolutionary 
of the vague concept of language by the faculty of language and the following introduction of the division between the faculty of language in the broad sense (FLB) and the faculty of language in the narrow sense (FLN), together with the idea that only that last concept precisely describes human cognitive abilities, set up well (or program well) the methodology of cognitive research, and whether the authors really pioneer the conceptual order by making these differentiations. Aiming so high, I will once again repeat focal questions: What indeed is the faculty of language?; How does this knowledge relate to other cognitive skills?; How serious were transformations within structure of this concept in the span between the publication of Aspects of the Theory of Syntax by Noam Chomsky and the publication of Simpler syntax by Peter W. Culicover and Ray Jackendoff (Chomsky 1965; Culicover \& Jackendoff 2005)? Ultimately, I would like to ask yet another question: what does this concept mean to us today that; is what engineering elements should we associate with it?

I intend to perform the above in three steps, or, should you prefer, in three acts. The first, strictly formal and conceptual, is to think over terminological problems, the sheer concept of the language faculty. The second step is to reflect on the structure of evolutionary explanations and the possibility of framing an answer to the question posed in the title - Has language evolved for other than language related reasons? Finally, in the step three, I will be pondering on the concept of adaptation and the usefulness of this concept in language research, referring both to the faculty of language in the broad sense (FLB) and the faculty of language in the narrow sense (FLN). I would like to underline that out of three questions put forth by Hauser, Chomsky and Fitch, the most interesting for me is the primary one: What is the faculty of language?

beginnings of language, but also tries to make of it a more systematic field of research and to define its program by determining conflicting points and points of possible consensus. The article started a debate that opened up with a critical response of Steven Pinker and Ray Jackendoff (Pinker \& Jackendoff 2005a), and continued with the answer of Fitch, Hauser and Chomsky (Fitch, Hauser \& Chomsky 2005) and the following retort of Jackendoff and Pinker (Jackendoff \& Pinker 2005b). It is rather amusing, that prior to 2002, Chomsky and Hauser had been on opposite sides on most issues. Hauser believed that language was on a continuum with animal communication and had emerged through natural selection. Chomsky believed language was totally distinct from animal communication and did not believe that language had been specifically selected for. Hauser, Chomsky \& Fitch represented a strategic compromise. Chomsky yielded to Hauser on most aspects of language but preserved what was most vital to him: a unique central process for syntax, one that had not been specifically selected for as a component of language, thus preserving intact his claim of uniqueness and independence from natural selection over a more limited domain. 
The obvious space limitation does not allow us to go deeper into the history of thinking about the concept of faculty of language. I will also spare the reader a reconstruction of reasons why this concept was introduced to psycholinguistics. Let me only remark that it was as late as in 1975 that Chomsky claimed in his Reflections on language:

The place of language faculty within cognitive capacity is a matter for discovery, not stipulation. The same is true of the place of grammar within the system of acquired cognitive structures. My own, quite tentative, belief is that there is an autonomous system of formal grammar, determined in principle by language faculty and its component Universal Grammar. This formal grammar generates abstract structures that are associated with "logical forms" ... by further principles of grammar. But beyond this, it may well be impossible to distinguish sharply between linguistic and nonliguistic components of knowledge and belief. Thus an actual language may result only from the interaction of several mental faculties, one being the faculty of language. There may be no concrete specimen of which we can say. These are solely the product of the language faculty; and no specific acts that result solely from the exercise of linguistic functions. (Chomsky 1975: 43)

Chomsky has never been enthusiastic about the concept of "language" and that made him opt for a more precise and fertile concepts of grammar and the language faculty. It is noteworthy that the conciliatory style employed by Chomsky, still present in the quoted work and suggesting a difficulty in precise separation of the language faculty from other cognitive powers, disappeared by 2002 with the publication of "The faculty of language: What is it, who has it, and how did it evolve?". Hauser, Chomsky \& Fitch suggest that not only the word "language" has divergent meanings depending on context and discipline; in informal usage it is understood as a culturally specific communication system, and in modern linguistics it is used in multiple ways to refer to an internal component of the mind/brain (sometimes called "internal language" or "I-language"). Their straightforward declarations are seemingly restrictive, e.g.: "We assume that 'internal language' is the primary object of interest for the study of the evolution and function of the language faculty" (Hauser, Chomsky \& Fitch 2002: 1570). However, there is more to it. Hauser, Chomsky and Fitch are in fact more solemn; for example, when they say that in exploring the problem of language evolution it is important to distinguish between questions concerning language as a communicative system and questions concerning computations underlying this system. 
Following their intuitive predictions, many acrimonious debates in the field of language evolution have been launched by a failure to distinguish between these problems.

Without going into details, their fundamental differentiation is: Faculty oflanguage in the broad sense (FLB) includes an internal computational system (FLN, below) combined with at least two other organism-internal systems "sensory-motor" and "conceptual-intentional." Hauser, Chomsky \& Fitch accept as uncontroversial the thesis of the existence of some biological capacity allowing humans (and not, for example, chimpanzees) to readily master any human language without explicit instruction. FLB includes this capacity but excludes other internal systems which are necessary but not sufficient for creation of language (e.g. memory, respiration, digestion, circulation, etc.).

In turn, Faculty of language in the narrow sense (FLN) is an abstract linguistic computational system on its own, independent of other systems that it interacts and interfaces with. It is important to understand that FLN is a component of FLB, and the mechanisms underlying it are a subset of those underlying FLB. A key component of FLN is a computational system (narrow syntax) which generates internal representations and maps them onto the sensory-motor interface through the phonological system and onto the conceptual-intentional interface through the formal semantic system. Hauser, Chomsky \& Fitch add that the core property of FLN is recursion. FLN takes a finite set of elements and yields a potentially infinite array of discrete expressions. This capacity of FLN yields discrete infinity, a property that also characterizes natural numbers. Each of these discrete expressions is then passed on to the sensory-motor and conceptual-intentional systems, which process and elaborate this information in language use. What is unique to our species is quite specific to FLN, and includes its internal operations as well as its interface with the other organism-internal systems of FLB.

Here, after the introduction of these subtle differentiations, which are intended to set us free from the vagueness of spoken language and to assure the authors' key declaration - namely that "internal language" is the primary object of interest for the study of the evolution and function of the language faculty - Hauser, Chomsky \& Fitch unexpectedly return to the meaning of the concept of language, saying that each expression is, in this sense, a pairing of sound and meaning; and further: "It has been recognized for thousands of years that language is, fundamentally, a system of soundmeaning connections" (Hauser, Chomsky \& Fitch 2002: 1571). It is very surprising as the whole idea of making the above differentiations was to set us free from the vagueness of concepts such as language, and what we are 
presented with is nothing else but its most intuitive definition. What else if not a combination of sounds and meanings is language for a language user?

Nonetheless, the question is why Hauser, Chomsky \& Fitch introduce the differentiation between FLN and FLB. What explanatory benefit do they promise? The reason I ask this question is that this form of explanation bears similiarity to the gnostic procedure of explaining dark with darker. The prima facie answer seems clear. Hauser, Chomsky \& Fitch say that approaching "language" as a monolithic whole confuses discussions of its evolution and blocks consideration of the useful sources of comparative data. However, there is a more productive approach which begins with unpacking FLB into its many component mechanisms. These components include both peripheral mechanisms necessary for the externalization of language and core linguistic computational/cognitive mechanisms. Each mechanism might have its own separate phylogenetic and functional history. FLN is restricted to a simple but powerful recursive mapping capability, which is by definition unique to humans and unique to the language faculty. This recursive mechanism has some plausible predecessors in other cognitive domains than that of communication. Thus, while accepting that FLB is an adaptation, Hauser, Chomsky \& Fitch hypothesize that FLN is not an adaptation "for communication." Is there no contradiction between these statements? Hauser, Chomsky \& Fitch insists that there is not, as long as the distinction between FLN and FLB is kept clear.

Still, I believe we should further investigate this issue. Is it really the case? Or maybe it is that Hauser, Chomsky \& Fitch - with their idea of dividing the language faculty into sub-mechanisms, each of them having separate evolutionary origin - create something that works only in conjunction and cannot be explained uniformly, even though it is useful for the sake of communication, and even if it does offer a research strategy capable of generating verifiable empirical hypothesis and credible explanations. Or maybe, the more plausible is the critique provided for by Ray Jackendoff and Steven Pinker, who challenge Hauser, Chomsky \& Fitch and accuse them of creating a dichotomy between language and non-language related capacities, as well as human and non-human cognitive abilities (Pinker \& Jackendoff 2005). According to Pinker and Jackendoff, the promised interdisciplinary approach ends up with a strategy that is in itself an isolating one, and which creates unwarranted dichotomies. Moreover, if FLN is a part of FLB, and if FLB is the form of adaptation "for communication," does it mean that the FLN element is not an adaptation with the aim of achieving the same goal?

Thus, we return to the questions raised in reference to the structure of the language faculty. The most important part of debate appears to centre around 
the computational apparatus underlying language, and especially syntax. Pinker and Jackendoff argue that syntax and other formal components of FLB are highly complex adaptations for communication, unique to language, and unique to humans, and thus that FLN is equally complex. They claim that syntax consists of a complex set of independent mechanisms whose interrelations and complexity are the distinctive signs of adaptation. If Hauser, Chomsky \& Fitch take the evolution of language seriously, they should also take seriously the possibility that no component of FLB may constitute adaptation for language, for communication or "for" anything at all, and this is as true of FLN as any other component.

Pinker and Jackendoff admit the following:

We agree that it is conceptually useful to distinguish between the language faculty in its broad and narrow sense, to dissect the broad language faculty into sensorimotor, conceptual, and grammatical components, and to differentiate among the issues of shared versus unique abilities, gradual versus saltational evolution, and continuity versus change of evolutionary function. ... Our disagreement specifically centers on the hypothesis that recursion is the only aspect of language that is special to it, that it evolved for functions other than language, and that this nullifies "the argument from design" that sees language as an adaptation. (Pinker, \& Jackendoff 2005: 204)

Hence, after such a declaration, we have the right to ask what this dispute is all about. What is the real object of this dispute? If Pinker and Jackendoff agree for the distinction of FLB and FLN, and Hauser, Chomsky \& Fitch agree that FLB is a highly complex adaptation for communication, then, again, what dispute is at stake here?

\section{Two kinds of similarity: analogous and homologous}

Without entering the dispute, I would like to spend a little while discussing the sole strategy of providing explanations in terms of evolution, and the possibility of creating of it a linguistic research strategy open to empirical verification.

It is tempting to think that if language evolved by gradual Darwinian natural selection, we should be able to find its predecessor in our closest relatives, the chimpanzee. In several famous demonstrations, chimpanzees have been taught hand-signs of American Sign Language, to manipulate 
coloured switches and tokens, and to understand a few spoken commands (Gardner \& Gardner 1969; Premack \& Premack 1983; Savage-Rumbaugh 1991). Is not really a scientific question if these abilities can be called language? In my opinion, it is rather a matter of definition, and our intention to classify how far we are willing to stretch the meaning of the word language or language faculty.

The proper scientific question is whether chimps' abilities are homologous to human language - and more precisely, whether these two systems show identical basic organization due to their descent from a single system in their common ancestors. For example, biologists are not debating if the wing-like structures of gliding rodents are called genuine wings or something else. Clearly, these structures are not homologous to bat wings, as they have a fundamentally different anatomical plan and reflect unique evolutionary history. Bat wings are hand modifications of a common mammalian ancestor; flying squirrel wings are modifications of the rib cage. The two structures are merely analogous: they share functional similarities.

The said distinction, which for us ought to be fundamental and binding, is widely applied within the field of biology, where we find two distinctive types of similarities - analogous and homologous. Analogous traits are the ones that share similiar function but have grown on different branches of the evolutionary tree and are not, strictly speaking, "the same" organ. The wings of birds and bees are a textbook example; they are both used for flight and share this similiarity that anything which is intended for flight has to be built in that same way. Those two, however, appeared independently in the course of evolution and have nothing in common except their use in flight. Homologous traits, in contrast, may or may not have a common function. Since they have a common ancestry, they have a common structure that allows us to see both as "the same" organ. The wing of a bat, the front leg of a horse, the flipper of a seal, the claw of a mole, and the hand of a human all have very different functions, but they are all modifications of the forelimb of the mammalian ancestor, and as a result they share nonfunctional traits like the number of bones and the ways they are connected (Pinker 1994: 343).

The interesting question is whether human language is homologous to - biologically "the same thing" as - anything else in the modern animal kingdom. Discovering a similarity like sequential ordering is pointless, especially when it is found on a remote branch that is surely not ancestral to humans. Here, at first glance, primates are relevant. Imagine that a chimpanzee can be taught to produce real signs, to group and order them consistently to convey meaning, and to use them spontaneously to describe events. Does that prove that human ability to learn language evolved from 
chimp's ability to learn an artificial sign system? Of course not, any more than the wing of a seagul is a sign of provenience with that of mosquitoes. No resemblance between the chimps' symbol system and human language is a legacy of their common ancestor.

Searching for a homology, one would have to find a distinctive trait which emerges both in an ape symbolic communication system and human language, the one which is not only indispensable to communication but is likely to have emerged twice in the course of evolution; once in human natural evolution and again in the lab, when psychologists contrive this system to teach their apes. One could search for such a signature in development when testing ape progress from jargon babbling to first words, and then two-word sequences to the explosion of grammar. One could also look at grammar development, checking if apes invent or favour given specimen of nouns, verbs, inflections, X-bar syntax, roots and stems, sentence auxiliaries forming questions, or other distinctive aspects of universal human grammar. We could also look at neuroanatomy, checking for signs of control performed by the left region of the perisylvian cortex, grammar part in front, and dictionary part at the rear. This line of questioning, routine in biology since the nineteenth century, has never been applied to chimp signing, though one can make a good prediction of what the answer will be.

A trait present in non-human animals did not evolve specifically in human language, although it may be part of the language faculty and may play a decisive role in language processing. It is possible, of course, that this trait evolved independently in nonhuman animals and humans, and as analogs rather than homologs. This would indicate the possibility that a given trait evolved for the purpose of creation of the language in humans and another reason in comparative animal group. In cases where the comparative group is nonhuman primates, and especially chimpanzees, there is little plausibility of this evolutionary scenario. In any case, comparative data is critical to rendering this judgment.

How does that affect our considerations? Well, it may mean that the scepticism of Hauser, Chomsky \& Fitch towards the possibility of providing an evolutionary explanation to creation of the language faculty in the broad sense is to some extent justified. What we may expect here are not homologous but analogous similarities. It does not mean, however, that their thesis concerning the faculty of language in the narrow sense (FLN), namely that it is a strictly human cognitive component, and the thesis combining this cognitive power with recursion, and the thesis that faculty of language in the narrow sense is more prone to empirical verification, are all somehow substantiated. It is hard to understand why finding the path of evolutionary 
events for the construction of recursion or their analogies in the evolutionary past is supposed to make it a more empirical research hypothesis than, for example, finding the same path and those same analogies for vocal skills or sense of rhythm, or the ability to conceptualize events. To the contrary, I daresay that the hypothesis of the narrow language faculty including only recursion is, in the light of the above differentiation, a sheer declaration of faith and expression of anthropological program, where human freedom and the ability to create advanced cognitive structures is a fundament of humanity.

Additionally, if recursion could have evolved in other animals "to solve other computational problems such as navigation, number quantification, or social relationships," then what is its relation to language or the language faculty? What is it that allows us to treat it as a part of the language faculty? This question is even more critical, as we are prone to accept that protolanguage had more to do with semantics than syntax, and that syntax is more of a late effect of the evolution. ${ }^{2}$ In this view, it is plausible that the capacity for syntactic structure evolved as an adaptive means of making such communication more informative and efficient.

On the other hand, the proposal of Pinker and Jackendoff is simply an expression of different research methodology and a portrayal of what they understand by the language faculty. Their objection to Hauser, Chomsky \& Fitch results from their disbelief that division between syntax, semantics, and phonology is accurate, and that the language faculty is built of the three elements combined. Pinker and Jackendoff demur from some of classificatory dichotomies of Hauser, Chomsky \& Fitch. These include (1) the Narrow/Broad dichotomy, which makes space only for completely novel capacities and for capacities taken intact from nonlinguistic and nonhuman capacities, omitting capacities that may have been substantially modified in the course of human evolution; (2) the current-utility/original-function dichotomy, which conceals the possibility of capacities that are adaptations for current use; (3) the human/nonhuman dichotomy, which fails to distinguish between independently evolved analogous functions and similarity due

2 This view on the evolutionary order of different linguistic functions stands in sharp contrast to mainstream contemporary linguistics. For followers of the Chomskian school, syntax is the primary object of study in linguistics; semantic features are added when grammar is not enough; and pragmatics is a wastebasket for what is left over (context, deixis, etc). However, when the goal is to develop a theory of the evolution of communication, the converse order - pragmatics before semantics before syntax - is more appropriate. In other words, there is much to find out about the evolution of communication, before we can understand the evolution of semantics and syntax. 
to inheritance from a recent common ancestor; and (4) the core/noncore and syntax/lexicon dichotomies, which omit the vast set of productive linguistic phenomena that cannot be analyzed in terms of narrow syntax, and which thus incorrectly isolate recursion - as the only unique development in the evolution of language (Jackendoff \& Pinker 2005: 224).

I would like to once again emphasise that in the opinion of Hauser, Chomsky \& Fitch, there is no question that language, appeared as a result of evolution, and that it is very useful to humans for variety of reasons. Hauser, Chomsky \& Fitch say that it shows signs of adaptive design, and to figure out the details of this evolutionary process will require comparative data and interdisciplinary cooperation. Hauser, Chomsky \& Fitch only discuss two aspects, first addressing the current utility of language, then turning to its past functions. Questions about current utility are empirically testable. But questions about original function of language are of a different logical type. It is rather unfortunate that the two main sources of data to address these historical issues, namely paleontological and comparative, are simply unavailable for behavioural traits unique to one species. Some behavioural traits are reflected in fossil data available to test hypotheses (e.g. we know from fossils that humans adopted a bipedal posture before brain size expansion), and for some linguistic mechanisms there may be relevant comparative data (e.g. for vocal learning). But, considering language as an unfractionated whole, neither type of data is available: "language" does not fossilize and it is unique to humans. Thus, from the empirical perspective, there are no and probably never will be data capable of discriminating among the many speculations that have been offered to define original functions of language, as for music, mathematical reasoning or a host of other interesting human abilities.

Empirically addressing specific hypotheses concerning adaptation requires equally specific hypotheses concerning function. "Communication" is far too vague a concept to constitute such a hypothesis, and none of the other candidates on offer seem much better. So why argue about them - ask Hauser, Chomsky and Fitch. Consider the analogous question: "What is the brain for?". No one would question the assertion that brain is an adaptation in some broad and not particularly helpful sense, but it would seem senseless to demand that neuroscientists agree upon an answer before studying neural function and computation. Even more specific questions like "what is the cerebellum for?" have defied resolution for many decades without blocking detailed and productive empirical research on this neural subsystem. The question "what was the cerebellum originally for?" is hardly even a topic of discussion. This is not to deny the possible utility of adaptive hypotheses 
in guiding empirical research: suitably specific adaptive hypotheses can serve a useful function in focusing and inspiring empirical research. However, at present there is no need for researchers interested in biology and the evolution of language to resolve these issues, or even take a stand on them. Such is the point of view of Hauser, Chomsky and Fitch (Fitch et al. 2005: 185).

This is exactly what tells apart the proposal of Pinker and Jackendoff and the one of Hauser, Chomsky \& Fitch. Pinker and Jackendoff are concerned with the question of what an adaptation is "for." To them, it seems quite obvious that "language is an adaptation for communication." They agree: it is true, we may not know whether bat echolocation is for navigating or for finding food, but we certainly know it is not for oxygenating the blood or nourishing the embryos. Our knowledge about its function has to be reframed at a more generic level, something like sensing the location and motion of objects in the dark. Likewise, it seems odd to claim that because we do not know whether primate vision evolved to find mates or food, we cannot even say anything about the adaptive function of the visual system (Jackendoff \& Pinker 2005: 213). ${ }^{3}$

Returning to language, Hauser, Chomsky \& Fitch suggest that the question "what is FLB for?" has clearly many answers if interpreted in terms of current utility. Today, FLB is used extensively in communication, private thought, in mathematics, logics, music, and in computer programming. According to Hauser, Chomsky \& Fitch better defined are the questions concerning specific current utility of FLN. This is my foremost reservation; I doubt whether the definition of FLN is so current, useful, straightforward and clear. I agree that the current utility of recursive mental operations is not limited to communication; recursive operations have clear utility for cognitive functions like interpreting mathematical formulas that are not plausibly adaptations at all, recursive thought would appear to be quite useful in such functions as planning, problem solving, or social cognition

Homo sapiens is the species with a symbolic language. According to the evolutionary theory, there should be a selective advantage fostering the development of language among humans. There are many explanations of such an evolutionary force. Some of the major ideas have stated that (1) language brings with itself the ability to convey information about prey or other food or about dangers of all sorts; (2) language is a result of sexual selection; (3) language replaces the social grooming found in monkeys and apes as an instrument for building coalitions and other social bonds; (4) language is a mother tongue that evolved among kin for honest communication; (5) language makes it possible to plan future action (Peter Gärdenfors 2003). However, despite all the merits of these proposals, they face a problem of explaining why language has not evolved among other apes or animals. I do not also claim that there is a unique explanation for why language has evolved among humans. 
that might themselves be adaptations. What the problem seems to be, is that no matter how often we mention how useful it is, it is hard to see any qualitative progress in comparing utilities ascribed to language. The sheer volume of the utilities we can provide for language is no greater that the volume of the utilities for recursion seen as adaptation! ${ }^{4}$

Bluntly speaking, I do not suppose that we are able to settle this dispute empirically, and I do think that what we have here is two competitive research programs, competitive as to organization and interpretation of available empirical data, but not their hypotheses. It does not mean, however, that the strategy of evolution focused research is infertile or tautological, as Chomsky has suggested in a couple remarks, e.g.:

It is perfectly safe to attribute this development [of innate mental structure] to natural selection, so long as we realize that there is no substance to this assertion, that it amounts to nothing more than a belief that there is some naturalistic explanation for these phenomena. In studying the evolution of mind, we cannot guess to what extent there are physically possible alternatives to, say, transformational generative grammar, for an organism meeting certain other physical conditions characteristic of humans. Conceivably, there are none - or very few - in which case talk about evolution of the language capacity is beside the point (Chmosky 1972: 97). ${ }^{5}$

4 For Hauser, Chomsky \& Fitch, the question is not whether FLN is adaptive in toto. By allowing us to communicate endless variety of thoughts, recursion is clearly an adaptive computation. The question is whether particular components of the functioning of FLN are adaptations for language, specifically acted upon by natural selection - or, even more broadly, whether FLN evolved for reasons other than communication? If, however, one entertains the hypothesis that recursion evolved to solve other computational problems such as navigation, number quantification, or social relationships, then it is possible that other animals have such abilities. If we find evidence for recursion in animals but in a noncommunicative domain, then we are more likely to pinpoint the mechanisms underlying this ability and the selective pressures that led to it. This discovery, in turn, would open the door to another suite of puzzles: Why did humans, but no other animal, take the power of recursion to create an open-ended and limitless system of communication? Why does our system of recursion operate over a broader range of elements or inputs (e.g., numbers, words) than other animals? One possibility, consistent with current thinking in the cognitive sciences, is that recursion in animals represents a modular system designed for a particular function (e.g., navigation) and impenetrable with respect to other systems. During evolution, the modular and highly domain-specific system of recursion may have become penetrable and domain-general. This opened the way for humans, perhaps uniquely, to apply the power of recursion to other problems. This change from domain-specific to domain-general may have been guided by particular selective pressures, unique to our evolutionary past, or as a consequence (by-product) of other kinds of neural reorganization.

5 There are other statements Chomsky made on the subject: "Evolutionary theory is informative about many things, but it has little to say, as of now, of questions of this nature 
This way, we approach one final issue I would like to investigate. In the end, I would like to ask whether the explanation based on natural selection is devoid of substance, meaning that there is only a "belief that there is some naturalistic explanation for these phenomena."

\section{Evolution, natural selection and adaptations}

The foremost thing we need to always keep in mind, is that the power of the theory of natural selection is that it connects two independent ideas. The first idea is the appearance of design. By appearance of design we mean something that an engineer could look at and surmise that its parts are shaped and arranged so as to carry out some function. Natural selection explains how this design came to be, using a second idea: the actuarial statistics of reproduction in the organism's ancestors. If we take a look at the two ideas: (1) A part of an organism appears to have been engineered to enhance its reproduction and (2) That organism's ancestors reproduced more effectively than their competitors, we will be able to note that (1) and (2) are logically independent. They are about different things: engineering design, and birth and death rates. You can say that an organism has good vision and that good vision should help it reproduce (1), without knowing how well that organism, or any organism, reproduce themselves.

The theory of natural selection says that (2), the ancestors' birth and death rates explain (1), the organism's engineering design - so it is not circular in the least and it is more - to use Chomsky's words - than "a belief that there is some naturalistic explanation for these phenomena." In fact, it is not so easy to show that a given trait is a product of selection. The trait has to be hereditary, it has to enhance the probability of reproduction of the organism, relative to organisms without this trait, in an environment like

[e.g., the evolution of language]. The answers may well lie not so much in the theory of natural selection as in molecular biology, in the study of what kinds of physical systems can develop under the conditions of life on earth and why, ultimately because of physical principles" (Chomsky 1988: 167). And just one: "It does seem very hard to believe that the specific character of organisms can be accounted for purely in terms of random mutation and selectional controls. I would imagine that the biology of a 100 years from now is going to deal with the evolution of organisms the way it now deals with the evolution of amino acids, assuming that there is just a fairly small space of physically possible systems that can realize complicated structures. Evolutionary theory appears to have very little to say about speciation, or about any kind of innovation. It can explain how you get a different distribution of qualities that are already present, but it does not say much about how new qualities can emerge" (Chomsky 1982: 23). 
the one its ancestors lived in. There has to be a sufficiently long lineage of similar organisms in the past. And because natural selection has no foresight, each intermediate stage in the evolution of an organ must confer some reproductive advantage on its possessor. Darwin noted that his theory made strong predictions and could be easily falsified. All it would take is the discovery of a trait that showed signs of design but that appeared somewhere else than at the end of a lineage of replicators who could have used it to help replication (Pinker 1994: 235).

This is of course in no way a settlement of dispute, nor an argument in favour of Pinker and Jackendoff against Hauser, Chomsky \& Fitch. It is only a warning, that it is too soon to discredit the evolutionary approach, as well as the doubts associated with it. It is only an attempt to exemplify how difficult it is to settle what is the precise object of academic disputes and how difficult it is for us to define our own stance, often accusing our interlocutors of their apparent misunderstanding and overinterpretation.

Attempting to give a straightforward answer to the question, I put forward in this paper - Has language evolved for other than language related reasons?, I would emphatically say - No, it has not!, as long as language has still anything to do with symbolic expression of a person's thoughts - that is, making them public, as well as symbolic exchange, which next to the exchange of goods, and next to the exchange of women, is, according to antropologists, the cornerstone of our culture. But if we understand language as a system of internal computations in a living organism, which are based on recursion and resemble a prototype of general intelligence, and which can be used in the domain of social exchange of goods, perception of kinship, communication, counting livestock, navigation, and setting order in a sentence, then I would have to affirmatively say - Yes, it has! Do these skills have anything to do with language seen as a means of communication or language seen as a paring of sound and meaning? Well, probably no more than the exchange of goods has to do with the exchange of symbols. I draw the general conclusion: in my initial question - Has language evolved for other than language related reasons? - the most important concept is not that of evolution or the faculty of language, but what I call "language related reasons," as it means anything we may wish it to, depending on what is meant by "language." 


\section{References}

Chomsky, N. (1965). Aspects of the theory of syntax. Cambridge, MA: MIT Press.

Chomsky, N. (1972). Language and mind. New York: Harcourt, Brace, and World. (Extended edition).

Chomsky, N. (1975). Reflections on language. New York: Pantheon.

Chomsky, N. (1982). Noam Chomsky on the generative enterprise: A discussion with Riny Junybregts and Henk van Riemsdijk. Dordrecht: Foris.

Chomsky, N. (1988). Language and problems of knowledge: The Managua lectures. Cambridge, MA: MIT Press.

Culicover, P. W., \& Jackendoff, R. (2005). Simpler syntax. New York: Oxford University.

Fitch, T., Hauser, M. \& Chomsky, N. (2005). The evolution of the language faculty: clarifications and implications. Cognition. 97(2), 179-210.

Gärdenfors, P. (2003) How Homo became sapiens: On the evolution of thinking. Oxford: Oxford University Press.

Gardner, R. A. \& Gardner, B. T. (1969). Teaching sign language to a chimpanzee. Science, 165, 664-672.

Hauser, M. D., Chomsky, N., \& Fitch, W. T. (2002). The faculty of language: What is it, who has it, and how did it evolve?. Science, 298, 1569-1579.

Jackendoff, R. \& Pinker, S. (2005). The Nature of the language faculty and its implications for evolution of language (Reply to Fitch, Hauser, \& Chomsky). Cognition 97 (2), 211-225.

Pinker, S. (1994) The language instinct. New York: Morrow.

Pinker, S. \& Jackendoff, R. (2005). The Faculty of language: What's special about it? Cognition 95 (2), 201-236.

Premack, D. \& Premack, A. J. (1983). The mind of an ape. New York: Norton.

Savage-Rumbaugh, E. S. (1991). Language learning in the bonobo: How and why they learn. In N. A. Krasnegor, D. M. Rumbaugh, R. L. Schiefelbusch, R. L., \& M. Studdert-Kennedy (Eds.) Biological and behavioral determinants of language development. Hillsdale, NJ: Erlbaum. 